\title{
Concordant Intestinal Atresia in Two Pairs of Monozygotic Twins
}

\author{
Mario Giuffrè, M.D., ${ }^{1}$ Mariavalentina Catania, M.D., ${ }^{1}$ and Giovanni Corsello, M.D. ${ }^{1}$
}

Intestinal atresia in both twins from the same pregnancy is very rare. Only seven pairs of twins have been described. The authors report on two cases of monozygotic twins with different types of intestinal atresia and clinical evolution. Both pairs of observed twins turned out to be concordant for the presence of intestinal malformations and for the absence of other linked malformations; nevertheless, the atresic lesions were anatomically discordant in each pair of monozygotic twins. Therefore, the diagnostic and therapeutic procedures have shown some differences in phenotypic expression between the twins of both pairs. Possible etiologic factors and pathogenetic pathways are discussed, and the importance of an accurate clinical and instrumental investigation and a long-term followup is underlined. Very rare models, such as pairs of monozygotic twins presenting intestinal atresia, represent an extraordinary resource to add new clinical and laboratory information likely to be useful in future advancements to understand the underlying etiology and pathogenesis.

KEYWORDS: Intestinal atresia, monozygotic twins, apple peel, double-bubble sign, malformation, concordance

Intestinal atresia is related to the absence of a normal opening of the gut or failure of structure to be tubular and is associated with narrowing or occlusion of the internal lumen. It is a leading cause of neonatal intestinal obstruction in developed countries and one of the most common causes of alimentary tract obstruction in the newborn, second only to anorectal malformation. ${ }^{1}$ Jejunoileal atresia occurs about once in every 5000 live births, and several cases of recurrence in the same family have been reported. Newborns from multiple pregnancies have a higher rate of small intestinal atresia than singletons, possibly due to vascular disruptions in monozygotic twins. Reports of intestinal atresia in both twins from the same pregnancy are very rare; only seven pairs of twins have been described in the literature. ${ }^{2}$ In

${ }^{1}$ Dipartimento Materno Infantile, Università degli Studi di Palermo, Palermo, Italy.

Address for correspondence and reprint requests: Mario Giuffrè, M.D., Dipartimento Materno Infantile, Università degli Studi di Palermo, Via Alfonso Giordano, 390127 Palermo, Italy (e-mail: giuffre@unipa.it).

Am J Perinatol Rep 2011;1:77-82. Copyright (C) 2011 by Thieme a 9-month period between 2007 and 2008, at the Policlinic of Palermo University, we observed two pairs of monozygotic twins with different types of intestinal atresia and clinical evolution.

\section{CLINICAL REPORTS}

\section{Patient 1A and 1B}

Two female twins (1A and $1 \mathrm{~B})$ were born from monochorionic biamniotic pregnancy at 31.4 weeks of gestation by elective caesarean delivery. Prenatal ultrasound evidence of polyhydramnios and "double-bubble" without other morphological abnormalities was obtained at 28 weeks of gestation for both fetuses. Macroscopic

Medical Publishers, Inc., 333 Seventh Avenue, New York, NY 10001, USA. Tel: +1 (212) 584-4662.

Received: December 3, 2010. Accepted after revision: April 14, 2011. Published online: July 1, 2011.

DOI: http://dx.doi.org/10.1055/s-0031-1281510.

ISSN 2157-6998. 


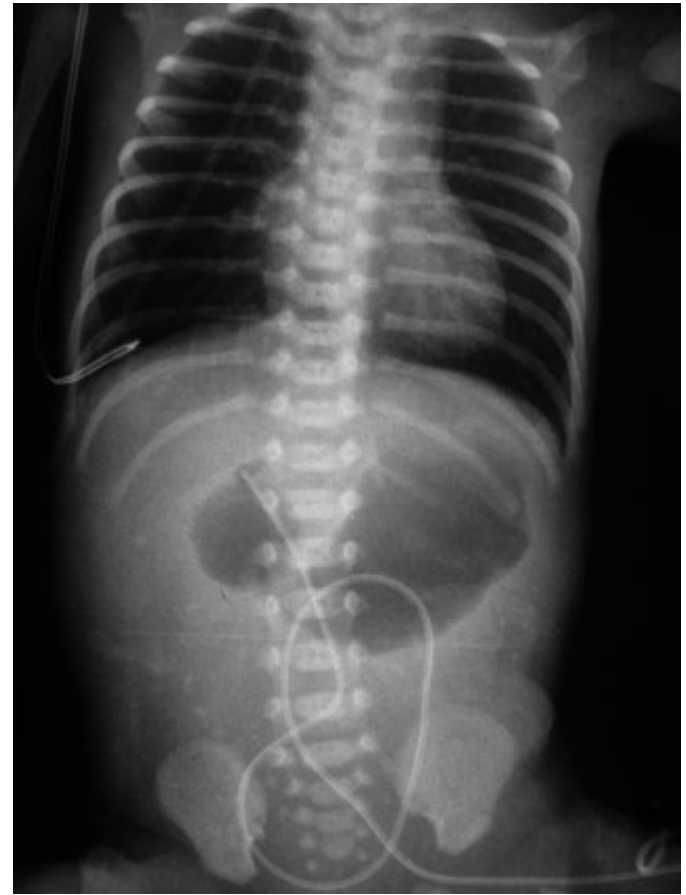

Figure 1 X-ray of patient $1 \mathrm{~A}$.

observation of placentas at birth did not show evidences of vascular accidents, but no histological investigation was performed. Apgar scores were $7 / 8$ for patient $1 \mathrm{~A}$ and 7/9 for patient $1 \mathrm{~B}$. Birth weights were 1330 and $1500 \mathrm{~g}$, respectively. An abdominal orthostatic X-ray showed abdominal distension and double-bubble sign in both twins (Figs. 1 and 2). A large amount of gastric fluid $(30 \mathrm{~mL}$ in patient $1 \mathrm{~A}$ and $50 \mathrm{~mL}$ of bilious gastric

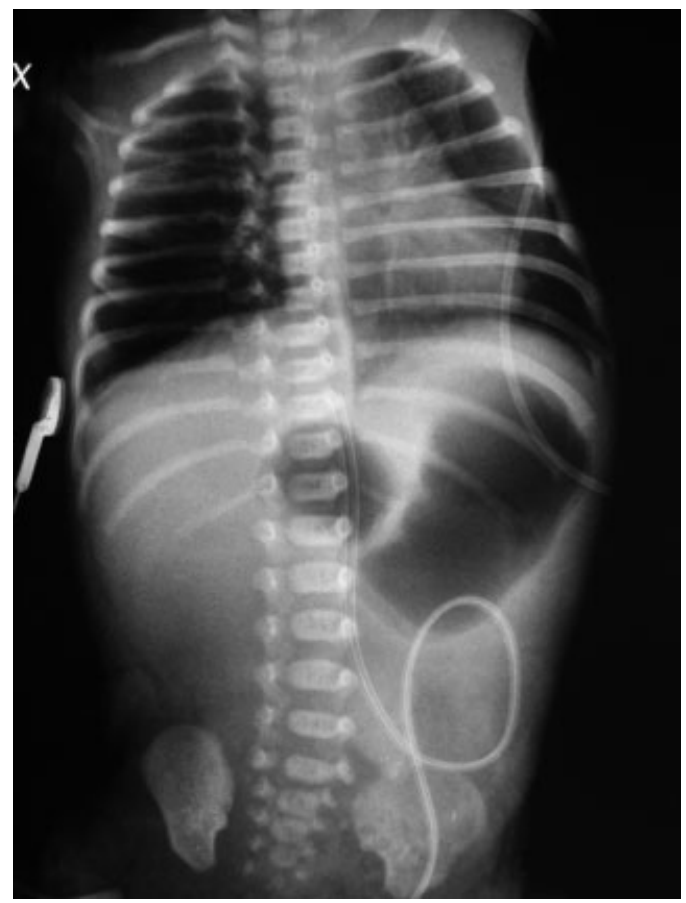

Figure 2 X-ray of patient 1B. residuals in patient $1 \mathrm{~B}$ ) were aspirated via the nasogastric tube. Therefore, enteral feeding was not started and total parenteral nutrition with central vascular access was provided.

Due to birth respiratory distress syndrome, both twins were intubated for 4 hours, followed by 48 hours of nasal continuous positive airway pressure. Phototherapy was performed on both patients from the third and second day of life, respectively, for $\sim 72$ hours.

Patient $1 \mathrm{~A}$ underwent laparotomy on the fifth day of life, which showed a proximal duodenal atresia, a dilated duodenum, and a complete mucosal duodenal membrane in its distal part. The papilla was placed in the intermediate dilated duodenum, which appeared as a large biliary cystic mass (Fig. 3). Therefore, the duodenal membrane was removed and an end-to-end anastomosis between the residual duodenum and the first jejunal loop was performed. In the proximal part, the duodenal atresic tract was opened and an end-to-end gastroduodenal anastomosis was performed. After 29 days of total parenteral nutrition, the patient gradually started enteral nutrition with milk formula, and partial parenteral nutrition was suspended at 45 days of life.

Patient $1 \mathrm{~B}$ underwent laparotomy on the fourth day of life, which showed intestinal atresia between the duodenum and the proximal jejunal loop and an "apple peel" atresia with mesenteric defect and intestinal malrotation (Fig. 4). Therefore, the atresic part was excised and a duodenojejunal anastomosis was performed. After 30 days of total parenteral nutrition, the patient gradually started enteral nutrition with milk formula, and partial parenteral nutrition was suspended at 40 days of life.

At 30 months after birth, an appendectomy was performed on patient $1 \mathrm{~A}$.

The babies are currently 32 months old. At follow-up, growth pattern appears adequate for patient

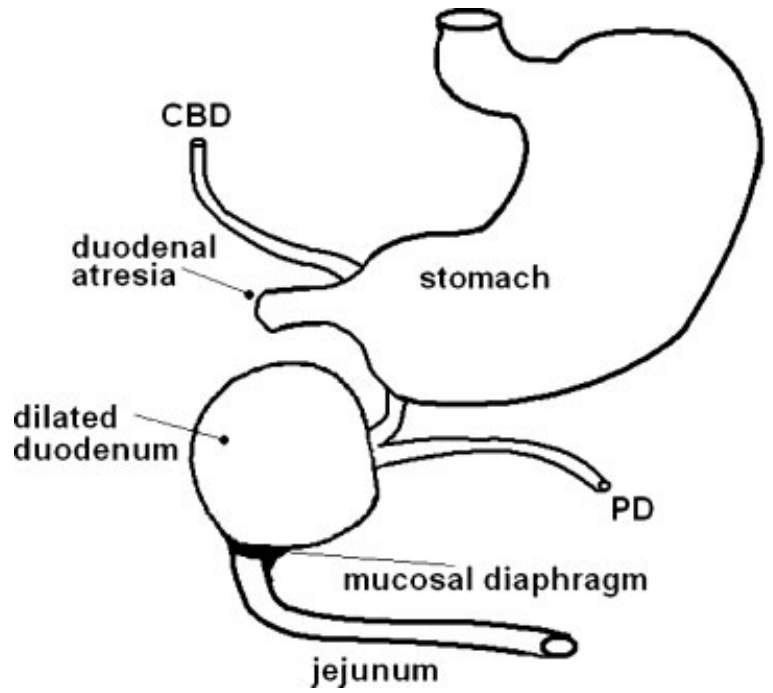

Figure 3 Scheme of intestinal malformation in patient $1 \mathrm{~A}$. CBD, common bile duct; PD, pancreatic duct. 


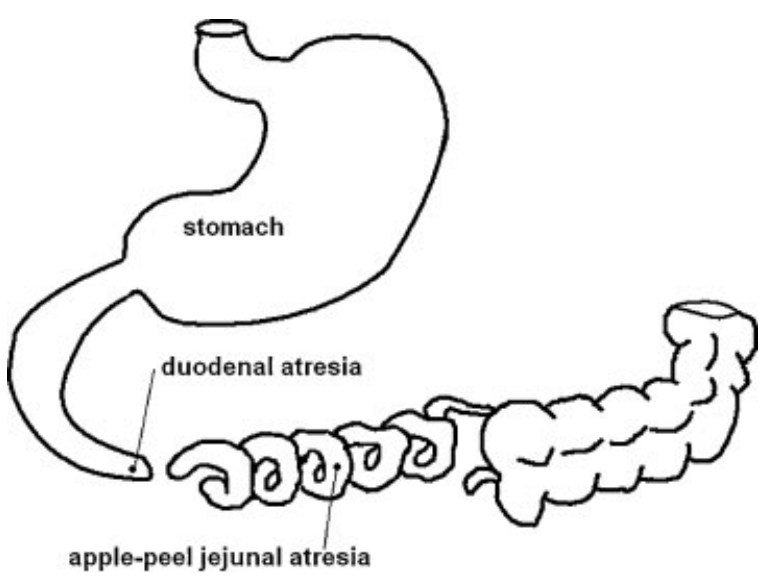

Figure 4 Scheme of intestinal malformation in patient $1 \mathrm{~B}$.

1B (weight, length, and head circumference at 50th percentile), but patient $1 \mathrm{~A}$ is 3 rd to 10 th percentile for weight, 10th to 25th percentile for length, and 10th percentile for head circumference.

\section{Patients 2A and 2B}

Two female twins (2A and $2 \mathrm{~B}$ ) were born from monochorionic biamniotic twin pregnancy at 32.4 weeks of gestation by emergency caesarean delivery for premature labor. During pregnancy, intestinal atresia in both twins was suspected at the fetal ultrasound scanning. There was no family history of either malformation or consanguinity. No exposure to radiations or drugs was noted during the pregnancy. Macroscopic observation of placentas at birth did not show evidence of vascular accidents, but no histological investigation was performed. Apgar scores were 7/8 for patient $2 \mathrm{~A}$ and $7 / 9$ for patient 2B. Birth weights were 1390 and $1550 \mathrm{~g}$, respectively.

Clinically, they showed abdominal overdistension; an abdominal orthostatic X-ray showed doublebubble sign in both babies. No other malformations were found. Phototherapy was performed on both twins from the second to sixth days of life. Both twins underwent surgical operation on the third day of life.

In patient $2 \mathrm{~A}$, the entire small intestine was atresic and malrotated (from almost the beginning of jejunum down to the distal part of the ileus only $5 \mathrm{~cm}$ before the ileocecal valve) and showed apple peel malformation (Fig. 5), therefore she underwent excision of the atresic intestine with proximal jejunostomy and distal ileostomy. Total parenteral nutrition was performed for 14 days, then she gradually started enteral nutrition with milk formula containing essential amino acids. At the age of 2 months and 7 days of life, patient $2 \mathrm{~A}$ underwent intestinal recanalization, totally measuring $20 \mathrm{~cm}$, and prophylactic appendectomy and cholecystectomy. Hepatic intraoperative biopsy showed alterations due to total parenteral nutrition, which progressively regressed after starting enteral feeding to

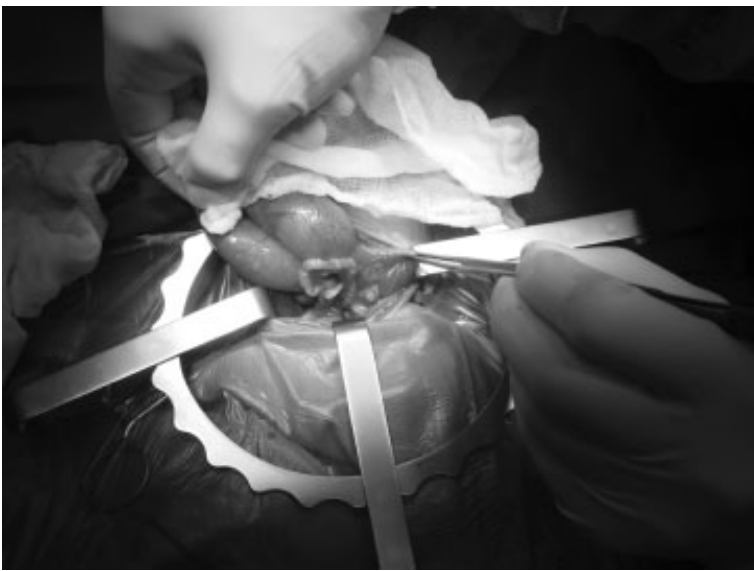

Figure 5 Apple peel malformation on patient 2A.

support partial parenteral nutrition. Neurological and ophthalmological screenings were normal.

At 7 months of age, central venous catheterization was performed on the right internal jugular vein with a nutritional schedule of $53 \%$ nocturnal parenteral nutrition and $47 \%$ daytime enteral nutrition. At 8 months of age, patient $2 \mathrm{~A}$ weighed $4900 \mathrm{~g}(<3 \mathrm{rd}$ percentile), her length was $62.5 \mathrm{~cm}$ (10th percentile), and head circumference was $37 \mathrm{~cm}$. At 14 months of age, she weighed $6320 \mathrm{~g}$, her length was $69 \mathrm{~cm}$, and head circumference was $43 \mathrm{~cm}$ (<3rd percentile).

Patient $2 \mathrm{~B}$ showed volvulus and apple peel malformation with meconium peritonitis and diffuse necrosis of the entire small intestine down to $1 \mathrm{~cm}$ before the ileocecal valve, and therefore she underwent excision of the atresic intestine with proximal jejunostomy and distal ileostomy (Fig. 6). The apple peel malformation caused a major loss of intestinal length. Postoperative recovery was associated with a prolonged period of intestinal inactivity, hence total parenteral nutritional was needed for 15 days followed by enteral feeding with milk formula containing essential amino acids supporting partial parenteral nutrition. At 3 months of age

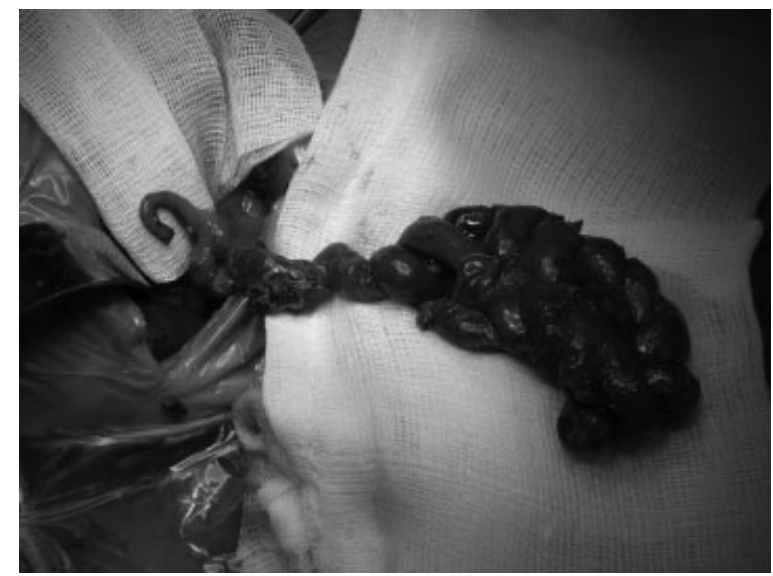

Figure 6 Apple peel with meconium peritonitis and diffuse necrosis on patient 2B. 
patient, $2 \mathrm{~B}$ underwent surgery for intestinal recanalization, after an intestinal elongation using serial transverse enteroplasty. Hepatic intraoperative biopsy showed alterations due to total parenteral nutrition. After surgery, patient 2B had a slow recovery of intestinal transit and episodes of diarrhea and regurgitations that required temporary interruption of enteral nutrition. Intestinal transit investigation showed an anastomotic jejunoileal stenosis treated with fluoroscopic pneumatic dilatations.

Despite positive enteral feeding tolerance, there was a progressive worsening of cholestatic indexes and splenomegaly; hepatic biopsy showed alterations due to total parenteral nutrition with portal hypertension and hepatic fibrosis. Therefore, lipids in parenteral nutrition were temporarily suspended and replaced by exclusive fish oil administration with progressive cholestatic indexes improvement, then lipids were gradually reintroduced.

At 8 months of age patient, patient $2 \mathrm{~B}$ weighed $4700 \mathrm{~g}$ ( $<3$ rd percentile), her length was $64 \mathrm{~cm}$ (25th to 50 th percentile), and head circumference was $40 \mathrm{~cm}$ (3rd to 10 th percentile). At discharge, she was fed on a nutritional schedule of $57 \%$ nocturnal parenteral nutrition and $43 \%$ daytime enteral nutrition. Her growth was still insufficient due to poor carbohydrate absorption, as showed by the xylose test, and her hepatic alterations worsened; therefore, carnitine was introduced in the parenteral nutrition. At 15 months of age, she weighed $5690 \mathrm{~g}$, her length was $66 \mathrm{~cm}$, and head circumference was $42.5 \mathrm{~cm}$ ( $<3 \mathrm{rd}$ percentile).

\section{DISCUSSION}

Intestinal atresia is a heterogeneous condition, with many etiologic factors and pathogenetic pathways involved, and several cases of familiar recurrence have been described. Different kinds and types of atresia, at different levels (duodenal, jejunal, ileal) and with different extension have been described. Type IIIB or apple peel atresia is a rare but well-know variant of jejunoileal atresia. In this birth defect, the distal ileum receives its blood supply from the ileocolic or right colic artery in a retrograde fashion. ${ }^{3}$ The apple peel deformity is usually found in premature infants and is associated with a significant loss of small intestine, physiologically short bowel, and subsequent feeding intolerance. ${ }^{4}$ Consequently, the defect is associated with high mortality, often resulting from inability to adequately provide nutrition for these children, though recent reports suggest an improvement in prognosis. ${ }^{5-7}$

Polyhydramnios and dilated intestinal loops, seen on antenatal scan, lead to suspected intestinal discontinuity. After birth, babies with atresia present failure to tolerate feeds, bilious vomiting, and abdominal distension. The classic double-bubble sign on abdominal X-ray may be the pathognomonic diagnostic marker.
Classically, there are two theories to explain the pathogenesis of intestinal atresia. Tandler supposed an error of the revacuolization of the solid cord of intestine, with a lack of recanalization in the early embryonic period. ${ }^{8}$ Then, Louw and Barnard presented their observation on the origin of intestinal atresia in the midgut. ${ }^{9}$ They proposed that intestinal atresia was caused by an embryological "vascular accident." There is no specific teratogen or genetic factor identified as a causal agent of either theoretic mechanism of development. Genetic factors or teratogen agents could cause a defect in the process of recanalization of the intestine as well as in the vascular supply to a segment of the fetal bowel. ${ }^{10}$ Familial hereditary forms of duodenal, jejunoileal, colonic, and multiple atresias have been reported. ${ }^{11,12}$ The associated presence of nongastrointestinal developmental defects raises the possibility of intestinal atresia being an autosomal-recessive heritable condition in some cases. It may also be associated to Down syndrome.

Recently, Fairbanks and colleagues evaluated the regulatory role of the fibroblast growth factor (Fgf) 10 and fibroblast growth factor receptor (Fgfr) 2b signaling pathway in the manifestation of intestinal atresia. ${ }^{13,14}$ The fibroblast growth factors act through tyrosine kinase transmembrane receptors. Fgfr $2 \mathrm{~b}$ is a receptor for Fgfs 1, 3,7 , and 10 . These receptor ligand interactions have a role in mesenchymal and epithelial cell interactions. The Fgfr gene family has genetic linkage to skeletal dysplasias, autosomal-dominant craniosynostosis syndromes, and mammary gland development, including gastrointestinal malformations. The involvement of the Fgf10/ Fgfr2b pathway in the pathogenesis of intestinal atresia is related to tissue-specific transcription factors, which regulate the expression of Fgf10 according to the organ system and the stage of gestation. ${ }^{15,16}$ Homozygous deletion of Fgfr $2 \mathrm{~b}$ in embryonic mouse permitted evaluation of the role of this signaling pathway in regulating proliferation and apoptosis in intestinal development; it results in a decrease in the rate of proliferation both in the mesenchyme and in the epithelium. The effect of deletion of Fgfr2b is a duodenal atresia with a $35 \%$ penetrance and a variable phenotype, without vascular malformation, raising questions about the role of vasculogenesis in intestinal atresia.

Seven sets of twins with jejunal or ileal atresia have already been reported in scientific literature: for one pair, there are no descriptive data available, and for all other pairs, most data are incomplete, important information is lacking, and there is no follow-up. All patients born before 1969 did not survive, whereas all patients born since 1980 did survive. Type I atresia and type IIIB atresia were the most frequently observed (five patients each). Only three pairs showed the same type of atresia in both twins. Only one patient showed an associated major malformation (gastroschisis), and in three patients, malrotation was described. 
The observation and the detailed description of two additional pairs of twins affected by intestinal atresia represent an important contribution to scientific knowledge. The present twins all survived the neonatal period and have a long-term follow-up (up to 3 years). The first pair showed a discordant type of atresia, and the second pair both showed type IIIB atresia but with different phenotypic expression and long-term outcome. The silent family and pregnancy history and the phenotypical differences between twins in each pair support the role of environmental factors in the pathogenesis. Nevertheless, genetic anomalies should always be considered in case of occurrence of similar malformations in both twins of monochorionic pregnancy.

\section{CONCLUSION}

Intestinal atresia, although rare, may be a significant clinical problem facing the neonatal patient population. The diagnosis may be made by prenatal ultrasound scan or after birth in the presence of bilious vomiting and the classic double-bubble sign on abdominal X-ray.

Only seven pairs of twins are described, and our cases are the eighth and ninth pairs of twins both affected by intestinal atresia. Mortality still remains high, and only seven neonates of these pairs of twins did not die in the neonatal period; therefore, we need long-term follow-up to learn more about the evolution of the natural history of these patients.

In our report, both pairs of observed twins turned out to be concordant for the presence of intestinal malformations and for the absence of other linked malformations. Nevertheless, the atresic lesions were anatomically discordant in each pair of monozygotic twins. Therefore, the diagnostic and therapeutic procedures have shown some differences in phenotypic expression between the twins of both pairs. Such differences may be negligible for the short- and longterm outcome (in example for the first pair of twins in this report) or may be significantly relevant (in example for the patient $2 \mathrm{~B}$, who required more sophisticated surgical treatments and still presents a more severe prognosis).

No chromosomal anomalies or mitochondrial diseases were found in these patients; possibly, an autosomal-recessive inheritance could be supposed.

Today, the paucity of available data does not allow to distinguish adequately the genetic and/or environmental factors causing intestinal malformations. Very rare models, such as pairs of monozygotic twins presenting intestinal atresia, represent an extraordinary resource to add new clinical and laboratory information to understand the underlying etiology and pathogenesis. These patients need an accurate clinical and instrumental investigation and a long-term follow-up that may reveal significant differences or new information likely to be useful in the next future advancements.

\section{REFERENCES}

1. Chirdan LB, Uba AF, Pam SD. Intestinal atresia: management problems in a developing country. Pediatr Surg Int 2004;20:834-837

2. Matsumoto Y, Komatsu K, Tabata T. Jejuno-ileal atresia in identical twins: report of a case. Surg Today 2000;30: 438-440

3. Shorter NA, Georges A, Perenyi A, Garrow E. A proposed classification system for familial intestinal atresia and its relevance to the understanding of the etiology of jejunoileal atresia. J Pediatr Surg 2006;41:1822-1825

4. Barakat NA, Maati SH. Nutritional and surgical management of short bowel syndrome in neonates. Res J Medicine \& Med Sci 2009;4:220-223

5. Federici S, Domenichelli V, Antonellini C, Dòmini R. Multiple intestinal atresia with apple peel syndrome: successful treatment by five end-to-end anastomoses, jejunostomy, and transanastomotic silicone stent. J Pediatr Surg 2003;38:1250-1252

6. Spencer AU, Neaga A, West B, et al. Pediatric short bowel syndrome: redefining predictors of success. Ann Surg 2005;242:403-409; discussion 409-412

7. Waldhausen JHT, Sawin RS. Improved long-term outcome for patients with jejunoileal apple peel atresia. J Pediatr Surg 1997;32:1307-1309

8. Tandler J. Entwicklungsgeschichte des Menschilichen Duodenum in Fruhen Embruonalstadien. Morphol Jahrb 1900; 29:187-216

9. Louw JH, Barnard CN. Congenital intestinal atresia; observations on its origin. Lancet 1955;269:1065-1067

10. Yokoyama T, Ishizone S, Momose Y, Terada M, Kitahara S, Kawasaki S. Duodenal atresia in dizygotic twins. J Pediatr Surg 1997;32:1806-1808

11. Bilodeau A, Prasil P, Cloutier R, et al. Hereditary multiple intestinal atresia: thirty years later. J Pediatr Surg 2004;39: 726-730

12. Lambrecht $\mathrm{W}$, Kluth D. Hereditary multiple atresias of the gastrointestinal tract: report of a case and review of the literature. J Pediatr Surg 1998;33:794-797

13. Fairbanks TJ, Kanard R, Del Moral PM, et al. Fibroblast growth factor receptor 2 IIIb invalidation-a potential cause of familial duodenal atresia. J Pediatr Surg 2004;39:872-874

14. Fairbanks TJ, Sala FG, Kanard R, et al. The fibroblast growth factor pathway serves a regulatory role in proliferation and apoptosis in the pathogenesis of intestinal atresia. J Pediatr Surg 2006;41:132-136; discussion 132-136

15. Kanard RC, Fairbanks TJ, De Langhe SP, et al. Fibroblast growth factor-10 serves a regulatory role in duodenal development. J Pediatr Surg 2005;40:313-316

16. Tatekawa Y, Kanehiro H, Nakajima Y. Duodenal atresia associated with "apple peel" small bowel without deletion of fibroblast growth factor-10 or fibroblast growth factor receptor 2IIIb: report of a case. Surg Today 2007;37:430-433 